CORRECTION

https://doi.org/10.1038/s41586-019-1351-8

\title{
Author Correction: Cyclin \\ D-CDK4 kinase destabilizes \\ PD-L1 via cullin 3-SPOP to control \\ cancer immune surveillance
}

Jinfang Zhang, Xia Bu, Haizhen Wang, Yasheng Zhu, Yan Geng, Naoe Taira Nihira, Yuyong Tan, Yanpeng Ci, Fei Wu, Xiangpeng Dai, Jianping Guo, Yu-Han Huang, Caoqi Fan, Shancheng Ren, Yinghao Sun, Gordon J. Freeman, Piotr Sicinski \& Wenyi Wei

Correction to: Nature https://doi.org/10.1038/nature25015, published online 16 November 2017.

In the gel source data for Extended Data Fig. 2b (page 7 of Supplementary Fig. 1 of the Letter) of this Letter, the label "IB: Cyclin A" should read "IB: Cyclin E". Figure 1 of this Amendment shows the original, correct Extended Data Fig. 2b and its corrected Source Data. The original Letter has not been corrected.

Extended Data Fig. 2b

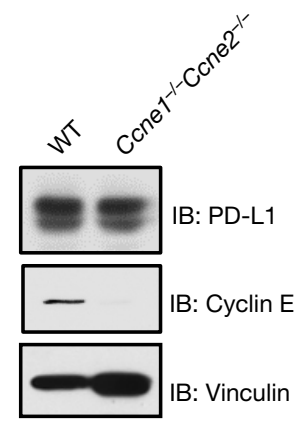

MEFs
Corrected gel source data for Extended Data Fig. 2b

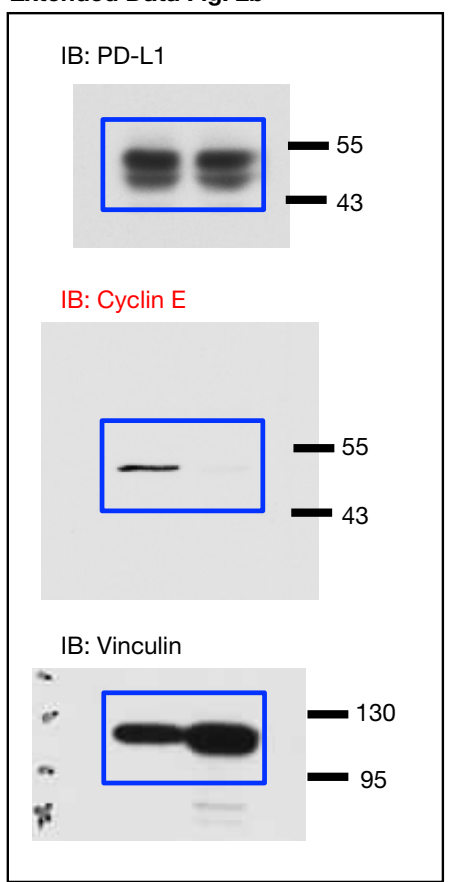

Fig. 1 This is the original, correct Extended Data Fig. 2b and its corrected Source Data. 